\title{
ON THE TRANSITION AND MIGRATION OF FLIGHT FUNCTIONS IN THE AIRSPACE SYSTEM
}

\author{
A. Terry Morris, NASA Langley Research Center, Hampton, Virginia \\ Steven D. Young, NASA Langley Research Center, Hampton, Virginia
}

\begin{abstract}
Since $\sim 400 \mathrm{BC}$, when man first replicated flying behavior with kites, up until the turn of the 20th century, when the Wright brothers performed the first successful powered human flight, flight functions have become available to man via significant support from man-made structures and devices. Over the past 100 years or so, technology has enabled several flight functions to migrate to automation and/or decision support systems. This migration continues with the United States' NextGen and Europe's Single European Sky (a.k.a. SESAR) initiatives. These overhauls of the airspace system will be accomplished by accommodating the functional capabilities, benefits, and limitations of technology and automation together with the unique and sometimes overlapping functional capabilities, benefits, and limitations of humans. This paper will discuss how a safe and effective migration of any flight function must consider several interrelated issues, including, for example, shared situation awareness, and automation addiction, or overreliance on automation. A long-term philosophical perspective is presented that considers all of these issues by primarily asking the following questions: How does one find an acceptable level of risk tolerance when allocating functions to automation versus humans? How does one measure or predict with confidence what the risks will be? These two questions and others will be considered from the two most-discussed paradigms involving the use of increasingly complex systems in the future: "humans as operators" and "humans as monitors."
\end{abstract}

\section{Nomenclature}
ATM Air Traffic Management
ATS Air Transportation System
FMS Flight Management System
GNC Guidance, Navigation and Control

NAS National Airspace System

NextGen Next Generation Air Transportation

System

SESAR Single European Sky ATM Research

SWIM System Wide Information Management

UAS Unmanned Aircraft System

\section{Introduction}

Migration, found in all major animal groups, is a behavioral adaptation that promotes survival and growth. The triggers for animal migration involve environmental factors such as climate and food. Throughout time, conditions have existed that either obligated the migration of a species, or permitted voluntary migration in whole or in part.

The same migratory phenomena can be observed in the way humans utilize the airspace. Instead of tracking movements of a particular animal species in a geospatial environment, this paper discusses how airspace functions have changed and been enabled by movements, or migrations, from nature (biology) to human-centered mechanisms, to collaborative human-automation systems, and eventually to automation-centered systems. Although the airspace may be utilized for many functions, such as communication and transportation mediums, this paper will focus on its use to enable transportation. The air transportation system (ATS) function expands and contracts, but is generally consistent across time - enabling an entity to move from an initial point to a destination via the airspace. Unlike the migration of animal species, the ATS function migrates in techno-political environments. Significant changes in the techno-political environments are one of the major causes of such migrations or transitions of function enablers. Other causes can be innovation, learning, technology advancements, and demand for new and improved services or performance. 


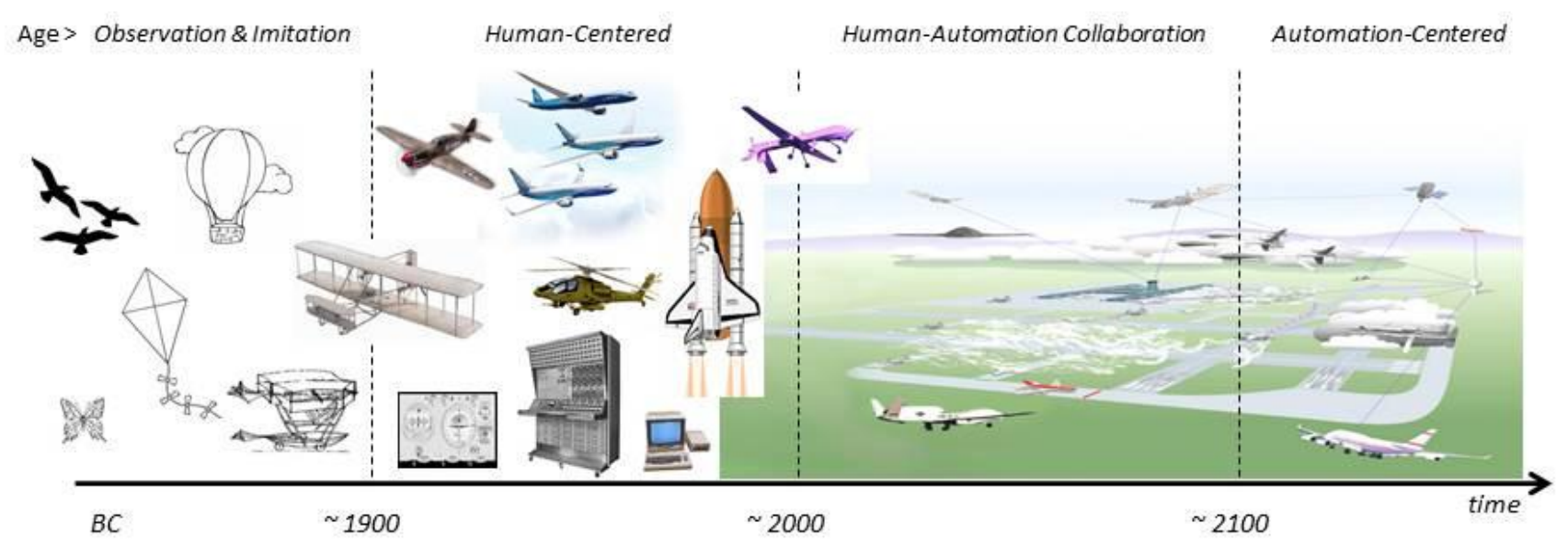

Figure 1. Ages of Air Transportation System Control

For this paper, transitions to new "migratory periods" occur when the nature of control over the ATS function undergoes a substantial change in authority (i.e. who is in control). In [1], this is referred to as the "locus of control." As previously mentioned, many conditions can trigger or affect these transitions. These include changes induced by the designer's paradigm, new means for ATS agent collaboration, or the addition of new agents who would be involved in control authority whether they be human or autonomous agents. Throughout each age, functions migrate almost continuously at a small scale, to satisfy the designers and the control authority within a techno-political environment. Despite undergoing such small changes continuously, there are four distinct migratory periods, or "ages," where a nearly steady-state control theme can be observed. These four ages of ATS control are denoted herein as: (1) Observation and Imitation, (2) Human-Centered, (3) Human-Automation Collaboration, and (4) Automation-Centered (Figure $1)$.

In recent years, the introduction and continued promotion of the Next Generation Air Transportation System (NextGen) in the US, and the Single European Sky ATM Research (SESAR) program in Europe, are pushing the global ATS function into increased use of automated and autonomous systems with integrated information networks that push system risks into new undiscovered areas [2]. These developments reinforce that we are currently in the early parts of the Human-Automation Collaboration age. This paper, targeted towards the community of ATS designers (e.g. architects, system safety specialists, technologists, policy makers, and certification authorities), among other things, raises questions about how to address safety during this and the next age - the Automation-Centered age. For example, how do we manage the sharing of responsibility for safety in ages where technological complexity has grown exponentially? Key aspects of the answer points to the importance of information and well-defined system requirements, as well as placing increased value on verification and validation of authority and autonomy boundary conditions, and on robust mitigations that can contain future risks given alternate roles for humans during design and operations.

\section{A. The Observation and Imitation Age}

The first age of ATS control was based on "observing and imitating." In this age, humans first observed nature, that is, the insects and the birds, and later imitated this flight via various mechanisms and techniques. For example, around $400 \mathrm{BC}$, the Chinese developed kites that could fly [3]. These kites were the forerunners of balloons and gliders all non-powered and dependent almost exclusively on nature (e.g. wind) to create lift. Leonardo daVinci in 1485 first illustrated and created ideas of man carrying machines, mimicking animal flight. The brothers Joseph and Jacques Montgolfier invented the first hot air balloon allowing the first manned flight in 1783. Around 1800, Sir George Cayley created a 
glider capable of carrying a human. In 1891, German engineer Otto Lilienthal designed a glider that could fly a person long distances. The cumulative effect of all such inventions, and the lessons-learned during the flights, led to the transition from the Observation and Imitation age to the Human-Centered age of ATS control.

\section{B. The Human-Centered Age}

The second age of ATS control began around the turn of the $20^{\text {th }}$ century and was embodied by a fundamental shift from the previous age in that controlled human flight was now possible. The advent of powered flight meant that humans were no longer at the mercy of natural forces, such as wind or gravity, to achieve flight. Thrust and directional control could be accomplished by human pilots with technology helping to accomplish this in cases where physical limitations existed. In 1901, Samuel Langley built the first successful flying aerodrome model propelled by an internal combustion engine that flew for nearly a mile. In 1903, the Wright brothers developed the "Flyer" with an engine propulsion system that allowed the first heavier-thanair human flight [4]. With this invention, humankind was now able to fly in the airspace with limited but effective control. The first armed airplane added functionality by incorporating a machine gun in 1912. Over time, innovators began to integrate electromechanical flight control systems to help human pilots control and manage aerodynamic performance (e.g. ailerons and elevators). This integration of flight-related functions also helped to efficiently optimize competing factors of performance and cost.

In 1914, the notion of automation was first introduced in the ATS when Lawrence Sperry's automatic gyrostabilizer led to the first "automatic pilot" [5]. Automation technology at this time added both functionality and complexity to the vehicle and suggested a possible replacement of human effort in the area of aircraft control. The first electromechanical flight simulator was introduced in 1928 by Edwin Link [6]. Boeing developed a commercial airliner in 1933. Over the next few decades, aircraft and ground-based innovations and technologies created many new and expanded ATS functions and capabilities (e.g. radar, instrument landing systems, radio-based navigation aids, and transponders).

Beginning in the 1950's, with the advent of the transistor and "digital" computer systems, analog functions began to be replaced. This allowed for more integrated functionality and savings in power and weight. Autonomous and automated systems continued to expand in function (e.g. auto-throttles, anti-skid braking systems, auto-landing, and automated decision support systems that alerted humans to changing conditions). The increased use of automated systems throughout the ATS as well as the increased utility of these systems incrementally but significantly changed the relationship between humans and automation. The human role in the ATS morphed as the automated system footprint expanded. In this age of ATS control, humans remained fundamentally in charge, although control (and authority) were beginning to be shared among the pilots and ground-based humans (e.g. air traffic controllers and airline dispatchers). In this age, humans are the designers and have ultimate authority. However, humans can decide whether or not to delegate this authority to automation under certain conditions.

\section{The Human-Automation Collaboration Age}

The third age of ATS control is initiated by a transition from human-centered control to a hybrid human-automation collaboration paradigm. Although aspects of the ATS involved such collaboration as early as the 1980's, the clearest indicator of this age can be seen in the development and promotion of NextGen and SESAR. NextGen is a transformation of the National Airspace System (NAS) in the U.S. It includes a transition to increased use of satellite-based technology, an informationcentric network infrastructure, and data link communications among aircraft and human/automated agents [2].

Both NextGen and SESAR are evolving the ATS through technological transformations and interoperability [7]. These transformations are transitioning airspace functions that were once the domain of ground personnel to ground-based computer systems and, in some cases, to the airborne systems. This transformation is also migrating functionality on the aircraft from the human pilot to computer-based automated systems. In early 2012, 
the US Congress passed a spending bill for the FAA allocating $\$ 64.3$ billion for modernizing air traffic control systems while expanding airspace access for unmanned aircraft systems (UAS) by 2015. This means that drones will have access to US airspace currently reserved for piloted aircraft [8]. The march towards increased human-automation collaboration is obviously well-underway [9].

\section{D.The Automation-Centered Age}

Though the far future is of course uncertain, projections of the current trend indicate that ATS control may eventually move from humanautomation collaboration towards an automationcentered control paradigm. Transition to the automation-centered age will occur when ATS designers, for any number of legitimate reasons, have demonstrated and validated the ATS as a nearly fully autonomous system that can have practically all control authority. This age will represent a major shift from today's small distinct cases of humandelegated authority (e.g. autopilot "modes") towards authority given to automation by design and irrespective of pilot or controller delegation during flight. This future age should not be feared as long as prudence, due diligence and sound decision-making are used by those involved in the design (e.g. architects, system safety specialists, technologists, policy makers and certification specialists).

Because airspace capacity and the communication spectrum are limited and the technopolitical environment can change dramatically, there are many uncertainties and risks associated with this future state. This paper will describe precautions that designers must address in order to reduce or control these uncertainties and risks. Effective requirements analysis and the verification and validation of the new authority and autonomy constructs are just two of the critical areas that designers must understand in order to deliberately create harmonious and riskmitigated ATS architectures that can enable this age.

\section{Impact of Technology Growth on Airspace Functions}

The ATS function can be decomposed by phase of flight into a set of basic sub-functions that include, for example, takeoff (lift, propulsion, control, navigation), fly in the airspace (guidance, navigation

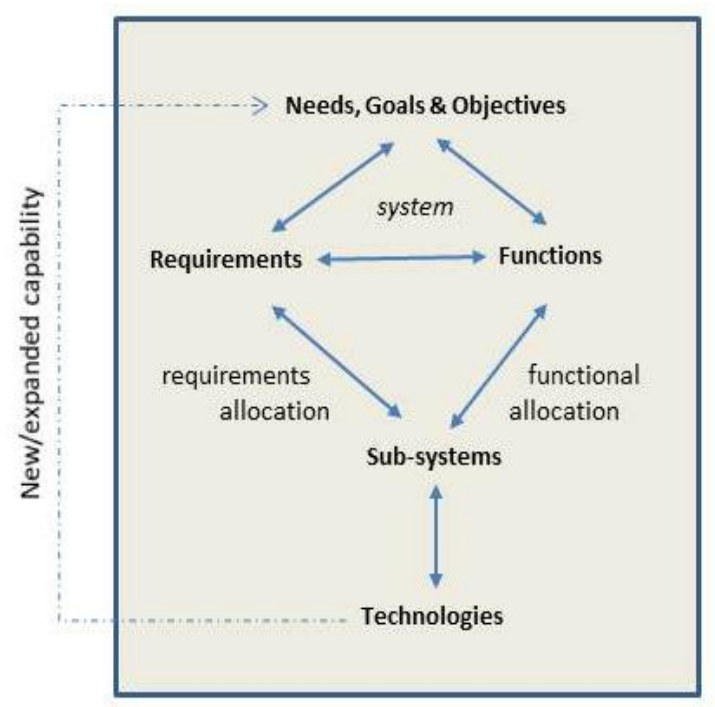

\section{Figure 2. Requirements, Functions and Technologies}

and control, propulsion), landing, and taxi. Many different types of technologies can be employed to aid in performing these sub-functions efficiently and safely. Using systems engineering processes, requirements are developed, and technology is applied as needed to meet the requirements. Required performance of the sub-functions may be achieved by either hardware, software, people or procedures. A growth in technology capability over the past 100 years or so has provided a means of realizing and optimizing ATS functions and achieving performance and safety that was previously unattainable.

\section{A. New Technologies Expand, Consolidate or Replace ATS Sub-Functions}

As with all technological progress, some technologies revolutionize while others allow for a more incremental and subtle evolution, with both leading to older technologies or methods becoming obsolete. Examples of technology impacts in the ATS include the development of more efficient airframe structures which revealed the aerodynamics of flight (weight, lift and thrust), balloon structures which led to gliders and then controlled flight, the development of steam engines which led to jet-propelled aircraft; improvements in thrust which led to faster aircraft with improved performance; and ground 
infrastructure efficiencies that permitted improved communication, awareness of weather and situational awareness of air traffic.

As new technologies become available, ATS designers look to improve efficiencies as long as safety can be maintained. New technologies typically reveal new capabilities that designers add to their systems thus allowing for new functions and an evolution of requirements (see Figure 2). Given the list of ATS sub-functions, new technologies have the potential to augment or expand, integrate or consolidate, overlap or altogether replace old technologies. As an example of technology that expanded ATS functionality, the aircraft industry from 1950 to 1970 improved engine technology from the piston-propeller to the turbo-propeller and eventually to the turbojet engine [10]. These technology transitions augmented and expanded airline mission profiles over time. Simple piston technologies are utilized on smaller aircraft and are well-suited for relatively short missions while the more powerful and complex turbine engines travelling at higher speeds and higher altitudes are used to fly longer distances.

As an example of how a substantially improved technology replaced predecessor technology, consider the transition to minicomputers in the ATS. Up to around 1961, vacuum tubes were used for ATS computing purposes. The first transition to transistor technology in 1962 resulted in much faster minicomputers. Within two years, integrated circuit technology provided astounding improvements over transistor technology [11]. This technology replaced its predecessor and allowed for expanded functionality in the ATS. Today, this form of technology assists or controls many of the ATS subfunctions. Improvements in this technology have led to autonomous and automated systems which can possibly even reliably perform tasks currently requiring human cognitive capabilities.

\section{B. The Limits of Technology and the Migration of Risk}

Despite the advantages provided by technology, all technologies have limitations. These limitations include the laws of physics, the combinatorial complexity of software when it comes to testing, trade-offs related to architectural design and function allocation, the implementation of non-functional requirements, economic considerations, integrating stakeholder inputs and political influence [12]. Technologies can also be hindered by developmental processes, certification, operational procedures and impediments from the organizational development team. Awareness of the pros and cons of technology must be considered - technology is not a panacea.

From a systems engineering perspective, each new technology integrated into the ATS impacts the system-level risk profile. Some technologies force the risk to migrate to different sub-systems while other technologies simply distribute the risk differently. This is because new technologies produce changes in system/sub-system functionality. Risks associated with these functional shifts, if unmitigated or poorly understood, may lead to unacceptable or unanticipated changes in system-level risk. Mitigations that were once satisfactory may be insufficient once a new technology replaces an older one. Awareness of risk migration caused by changes in technology should be a primary consideration and driver for those involved in ATS design [13].

\section{The Best of Both Worlds}

In the complex relationship between humans and automation, trade-offs are both necessary and challenging. This is particularly true when assigning roles, responsibilities and authority to human and/or automated agents. In [9], several design metaphors regarding these issues are described. Both humans and automation can co-exist in many different configurations, even within any of these design metaphors. According to the perspective provided in Figure 1, as we transition to new migratory ages, the ATS designers will have increased responsibility for safety and performance; while humans involved in operations (e.g. pilots) will have less responsibility/authority. As the transitions occur, clear, complete and consistent requirements; limitations and constraints for technology; and humans and system safety are essential considerations for the realization of to safe and effective designs.

\section{A. The Limits of Aviation Safety}

There are limitations to safety when the ATS relies on decisions from, and interactions between, humans and technology. Technology, as described 


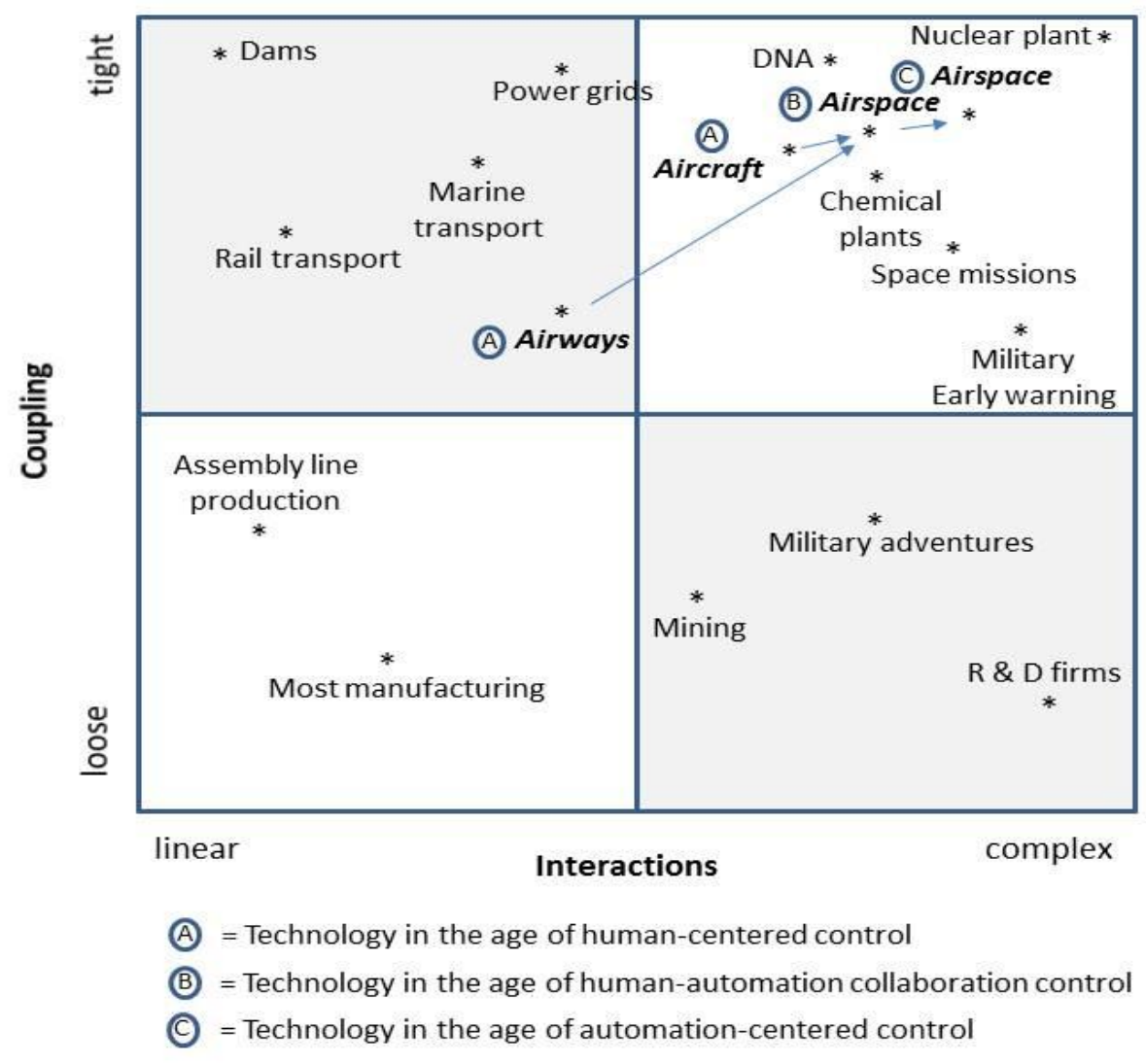

Figure 3. Technology Interaction/Coupling Chart

earlier, can be limited by laws of physics, testing complexity, fabrication/development constraints, cost, and even political will in some cases. Humans, in similar fashion, also have limits which can be cognitive and/or physiological. In the age of humancentered ATS control, the prevailing theme declares that pilots are the final authority for a flight and are responsible to monitor the aircraft and the environment and take corrective steps if automation or any other system malfunctions. In this role, human capabilities are often perceived as both the greatest constraint to safety and the greatest enabler of safety. Humans (in this case pilots and air traffic controllers) have decision-making authority that can directly determine the outcome of the flight. This sentiment remains true in the age of human-automation collaboration [14]. However, in the age of automation-centered ATS control, the limits of aviation safety will be determined almost solely by the human designers. In general, the limitations of the individuals and organizations with critical decisionmaking authority regarding design decisions will induce limitation on the ATS. Furthermore, almost all of the operational risk will move to designers, and in particular those involved in the verification and validation of designs.

Part of the reason these limits are manifest is the interactions and coupling of sub-systems within the ATS. Figure 3 shows the interaction/coupling technology chart developed by Charles Perrow [15]. According to Perrow, "aircraft" technologies are considerably more complex and tightly-coupled than "airway" technologies. Written in 1984, Perrow's classification of "aircraft" and "airway" was developed in the context of human-centered airspace control. By considering NextGen- and SESAR-like concepts that promote more complex technologies in the ATS, we can project where these advancements will lie on Perrow's mapping (Figure 3) and for the ages in Figure 1. As described by Perrow, the interaction between the elements of a device is considered complex if there are many alternative subtasks at any point in its execution. It is considered 
linear if it is comprised of a set of fixed steps carried out in sequence. The coupling dimension describes the extent to which an action is related to its consequences. Such a system is said to be tightlycoupled if consequences are linked closely with actions. In other words, minor slips in tightly-coupled systems can quickly become accidents. By contrast, in loosely-coupled systems, the link between an action and its consequences is less clear. These systems tend to be more forgiving of errors. These two dimensions form Perrow's interaction/coupling space with which technologies can be classified [16]. Using Perrow's initial placement for "aircraft" and "airway" for human-centered airspace control (items A in Figure 3), we consolidate both into an "airspace" technology data point which represents the increased coupling and complex interactions brought about by NextGen- and SESAR-like concepts. The item labeled " $B$ " represents the move to the age of human-automation collaboration while item "C" represents the move to the age of automationcentered ATS control. These estimated placements are sure to be adjusted as safeguards and redundancies are designed into future ATS architectures.

\section{B. The Automation Addiction Phenomenon}

Because of the way in which designers have prescribed the interaction between humans and automation, and their respective roles, a condition has emerged known as "automation addiction" [17]. Closely related to this phenomenon is complacency, which can be an outcome of automation addiction, and the level of engagement, which can be a contributing factor. Automation addiction occurs when pilots abdicate or delegate too much responsibility to automated systems, and over time, there is a weakening in their response effectiveness to emergencies or unexpected circumstances. Automation addiction, which is an over-reliance on automation, can also diminish effective use of electromechanical systems because human skills need to be practiced to prevent atrophy. Billings and others have referred to this condition as the "automation paradox" [18]. In the future where increased levels of automation are expected, designs will be even more vulnerable to this phenomenon. Effective mitigation will depend on understanding the dynamics of this automation addiction in order to impart more effective solutions.

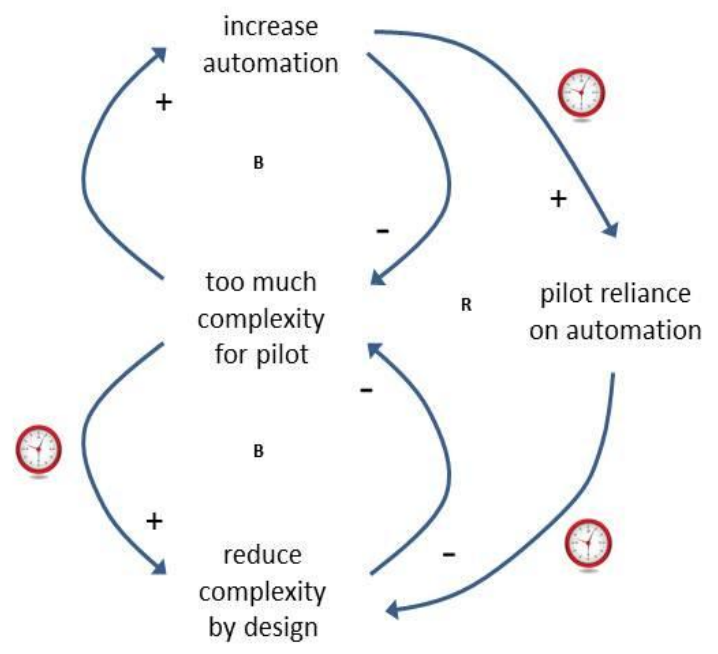

Figure 4. Dynamics of Automation Addiction

System dynamics models [19] utilize a variation of the shift the burden archetype to explain the dynamics and solutions to automation addiction [20]. Figure 4 depicts such a system dynamics model that represents the dynamics of automation addiction with humans. The arches represent positive ("+" increasing) or negative ("-" decreasing) influences between variables or objects. Clocks represent delays and " $\mathrm{B}$ " and " $\mathrm{R}$ " represent balanced and reinforcing loops, respectively.

Referring to Figure 4, the dynamics of automation addiction can be explained as follows. A problem exists for the pilot during a flight and is manifested as a symptom, or set of symptoms. The example shown is that there is too much information or complexity for the pilot to effectively perform a required task. This problem can be solved via multiple courses of action. One course of action, the symptomatic solution (i.e. increase automation) has an apparent advantage over a more fundamental solution (i.e. reduce complexity of the design) because of delays in facilitating a design change. As a result of too much information or complexity, automation is added to manage the complexity for the pilot and attempt to keep him aware of the situation using more abstracted feedback. A short-term symptomatic solution obviates the need to pursue the more fundamental solution of reducing complexity by design. However, the inability to work on the fundamental solution ensures that the problem symptom will return. 
Additionally, implementation of symptomatic solutions, in time, can create unintended side effects (e.g. a viscous cycle of increasing pilot reliance on automation) which over time works against implementing the fundamental solution of reducing complexity by design. Compounding this problem is that over time, as pilots rely more on automation, skills at non-automation supported tasks may atrophy [21]. This in turn places an added burden on training to reduce risk. The most obvious example of such a situation is the evolution of today's auto-flight systems, their various "modes," mode transitions, and the requisite enabling Flight Management System (FMS) and its interface.

When the dynamics of the addiction cycle are taken to the extreme, one of two possible conditions occur. First, application of symptomatic solutions leads the pilot to be totally dependent on the automation. The pilot, in this case, has unwittingly turned over control and authority to the automation via small incremental decisions. Second, application of the fundamental solution to reduce complexity by re-design leads to healthy, beneficial interaction between humans and automation. In either case, it is the designers that ultimately face the challenge of choosing a particular point design across this span of possible solutions.

\section{The Designer's Dilemma - Human Automation Functional Allocation}

As new technologies are developed and integrated in the ATS, it is incumbent on designers to architect systems that balance the risks and limitations of automation, technology and humans in order to achieve system safety and mission success goals. These decisions include the appropriate use of technology, the level of interaction between the human and automation, the degree to which safety can be impacted by hazards, faults, and desirable mitigations to control deleterious and unwanted behaviors. One of the primary means of architecting a system appropriately is first determining the needs, goals, and objectives (Figure 2) envisioned for the human, the automation, and the system as a whole. This step is also often called requirements development and analysis. One way of viewing human-automation design space is discussed in [1] and is based upon a two-dimensional space wherein one dimension is the "locus of control" and the other is "roles and responsibilities." In this construct, the degree to which humans and/or automation will provide functionality and control of the system can be represented at a high level. At the extremes of the "locus of control" axis are ground-based control and aircraft-based control; while the extremes of the "roles and responsibilities" axis are humans as a active operational decision-makers (e.g. pilots) versus humans as monitors/managers of systems during operations.

\section{Deliberate and Essential Designs}

Deliberate and essential designs are those designs where adequate forethought, analysis, and protections have been applied during design, development, and testing. Deliberate and essential designs strike an appropriate balance between humans and automation (thus reducing the likelihood of automation addiction) and regulate the flow of new technology into the system thus mitigating risks. One significant way of achieving deliberate designs is the use of a diverse set of designers who are comfortable exploring and analyzing in particular the uncertainties in the unknown unknowns [22].

There are many processes and tools (e.g. integrated hazard analysis [23]) that have been created to aid in producing deliberate and essential designs. Only two will be discussed here. They are effective requirements analysis, and verification and validation methods for authority and autonomy allocation.

Effective requirements analyses are necessary to create deliberate designs. Since the introduction of computing systems, much of the functionality of enabling airborne and ground-based systems is performed by the software. Raytheon found that approximately $40 \%$ of the total budget for their software projects was rework [24]. Bell Labs and IBM studies have determined that $80 \%$ of all product defects are inserted in the requirements definition stage [25]. In general, rework consumes 40 to 50 percent of software project budgets. This means that requirements errors can consume up to 42 percent of total software development resources. Therefore, effective requirements analyses reduces errors, improves safety, reduces costs and embody trade-offs necessary for balanced designs. Further, design tradeoffs have significant impact on the complexity of the overall system. For example, with respect to information management in the ATS, integrated 
versus segregated design trades impart coupling and interactions on the ATS infrastructure: segregated designs reduce coupling of information and increase redundancy, whereas integrated designs increase coupling between airborne and ground-based systems. This coupling tends to increase complexity due to data fusion and filtering that obscures raw sensor data.

Verification and validation of new authority and autonomy constructs will increase in importance as more ATS sub-functions transition from humancentered control to human-automation collaboration and eventually to automation-centered control. Critical verification and validation assurance methods must support designers in creating deliberate and essential designs. These methods must also uncover vulnerabilities that may otherwise result in accidents or unexpected behaviors. As NextGen- and SESARlike concepts mature and push the air traffic management toward more human-automation collaborative designs, the stress on verification and validation will grow to ensure the behavioral integrity of the collaborative aspects, particularly in situations where the collaboration may be between several human and automated agents. Such collaborative systems may be used for aspects of flight path management, four-dimensional trajectory negotiation and collision avoidance.

It is expected that verification and validation techniques can be leveraged in the commercial ATS from developments and experiences in the military sector. In the future, it is expected that military UAS operations will have a much larger portion of ATS operations. Automation and authority management will be taken more quickly into the automationcentric age. Verification and validation of these new constructs will be addressed here first and the lessons learned can then be transferred to the commercial sector as has been done many times in the past (e.g. the introduction of radar following World War II) [26].

In the age of automation-centered airspace control, the integrity of the system will rely on agile verification and validation processes to ensure the safety of crewed and unmanned aircraft. The push for expanded UAS use in civil airspace will drive the need for new and innovative verification and validation techniques to reduce system level risks.

\section{Accidental and Negligent Designs}

Accidental and negligent designs are designs that are developed haphazardly without much forethought into unintended consequences, or preventing unwanted system behavior. These system designs are characterized by negligence where faults and unsafe conditions have crept in due largely to carelessness, or a rush to product. Much of the human effort here is used to mitigate damage from unobservable system states caused by inadequate design practices. In a worse-case scenario, designers may have inadvertently created an environment where emergent sentient autonomous systems can gain airspace control. This and other deleterious conditions can be the result of negligence, but more often result from a lack of understanding of the complexity of the system and interactions between its components.

\section{Should the Pilot or the Designers Bear Ultimate Responsibility for Safety of Flight?}

In the current world of increasingly complex and disparate systems that enable the ATS, how does one determine whether the pilots or the designers should be held ultimately responsible for the safety of a flight? The answer of course depends on the context and purpose, or intended function, of the design. For example, in the human-centered age of ATS control, the pilot has primary responsibility for the aircraft and is the final authority. The designer has primary responsibility for the correct operation of the systems that support him. This remains the case, to date, in the age of human-automation collaboration, although the pilot can, and does, delegate authority to automation in many circumstances (e.g. auto-land). However, in the postulated future age of automationcentered ATS control, the designer will have ultimate responsibility for all aspects of safety. The human will have much less capability to intervene, or re-take authority.

\section{E. Designing for Humans as Operators}

The ATS control ages provide broad and discrete contexts by which to analyze the roles that humans and automation will perform. Humans, as operators of the ATS, form the basis for the age of human-centered ATS control, and this paradigm remains largely in place today, although the transition to the Human-Automation Collaboration age is also 
well underway. As human operators, pilots have authority for the aircraft while controllers and designers have authority over the airspace and systems that enable the ATS function. Given the active and engaged role of humans, designs are not only required for technological elements, but also the training and procedural requirements. For the ATS to be safe and effective, each of these designs must be well thought-out and thoroughly tested (i.e. a deliberate and essential design). Unfortunately, this has led to a very complex system of systems that is inflexible in many ways, and difficult to change beyond small incremental steps.

As we transition even more into the age of human-automation collaboration, human operators require a more intimate knowledge of the spectrum and dynamics of the automated systems that they are collaborating with. Humans will need to understand the technology limitations so that they may take control during times of uncertainty, or when the automation fails. Humans need to understand the inadequacies of automation to ensure that humans will be available, and trained, to operate in these system states. This includes the situation of both UAS and crewed aircraft procedures, mitigations and contingencies. Designers should have a sound understanding of the functional allocation (including overlap) between humans and automation in order to fully inform the operator during times of emergencies. Locus of control issues must be fully understood, and pilots/controllers should directly collaborate with designers during the design phase.

Finally, in the age of automation-centered ATS control, designers will hold primary responsibility for performance of the ATS. This will include automation that is flying the aircraft and controlling airspace access. Though humans will continue to operate as pilots here, they will have a much different role and we can expect much fewer in number relative to the number of aircraft (possibly due to an increase in UAS). Verification and validation techniques for complex systems of systems will need to reach an unprecedented level of sophistication to ensure safety and integrity.

\section{F. Designing for Humans as Monitors}

As stated earlier, in the age of human-centered ATS control, the human pilot has ultimate authority over the aircraft during operations. However, the pilot may, and does, delegate authority to automation in many circumstances. Once authority is delegated, the pilot is responsible for monitoring the performance of the automation to assure it performs its intended function and to reclaim authority should the automation fail. This system is extremely safe due to rigorous verification and validation processes and well-defined procedures and training. However, it has been shown that humans are not good at monitoring, in general. Therefore, vigilance to the task and active engagement is essential.

In the age of human-automation collaboration, the monitoring role will grow in many respects; however, there will be the added complexity of new modes of dialog and negotiation (i.e. collaboration). The delegation of authority to automation will be more fluid, frequent, and bi-directional [9]. Monitoring may involve consent to state changes and critical decisions, acknowledgements of system intentions and support for mutual awareness of faults, failures, or other conditions. Because humans may fluidly transition back and forth between operator and monitor, cognitive and mechanical flying skills will need to be honed and maintained in new ways. Designers in this age will need to have a full understanding of any and all state transitions, and include intuitive mechanisms for humans to observe these. Even closer relationships between the designers and the human monitors will be required to increase operational awareness and understanding.

In the age of automation-centered control, the predominant human function during operations will be that of a monitor for automation that will have authority for aircraft flight. The human in this scenario is in a predicament because it will be difficult to keep their flying skills current in an age that has little demand for this expertise. Human monitoring in this age will be "automation addiction" by design. In other words, we will be completely dependent on automation for many/most situations and conditions. In this age, pilots should have a close relationship with the designers who will have ultimate authority to constrain and exploit automation weaknesses if the need ever arises. In this age, one can envision a set of remote pilots who are available to step in under rare conditions where automation may have failed, but has failed to a safe state (e.g. a holding pattern). 


\section{Conclusions}

As the ATS continues to expand, functions that were once solely dedicated to humans are slowly migrating towards automated systems. This paper takes a broad philosophical perspective in defining ages of ATS control from prehistory, when mankind observed and imitated the flight of birds, to the current age of powered flight where pilots have authority for safety of flight. Looking now and into the near future, an age of human-automation collaboration is apparent wherein humans are still in control and have authority during flights, but several functions are delegated to automation, while others are collaboratively decided. In the far future, an automation-centered age can be anticipated where the ATS is designed to be largely automated and this same automation is given authority with regard to control decisions affecting safety. Collectively, these migratory ages and the transitions between them provide a set of contexts by which to analyze functions, either at a high-level conceptual scale, or at lower-level scales. From this perspective, ATS sub-functions have been continuously moving from nature to humans to autonomous systems. This functional migration is very similar to the migration of birds and bison in that survival is predicated on finding an environment that permits them to flourish. Birds and bison move in a geospatial environment whereas functions move in a techno-political one. Each step of the migration involves numerous individual and collective decisions by the designers. Further, it is the designers who will knowingly or unknowingly push the ATS into paths of sustainability (deliberate designs) or unsustainability (negligent designs) by the growth and infusion of technology in the ATS infrastructure. Awareness of these contexts can help designers discern beneficial trade-offs, despite human and technological limitations, as they prepare to take the ATS into unprecedented new territory.

\section{References}

[1] Rogers, Bill, 10-12 May 2011, "NextDeck: Flight Deck Automation Designs for NextGen," Proceedings of the NASA Aviation Safety Program Annual Technical Meeting, St. Louis, MO.
[2] NextGen Implementation Plan, March 2012, Federal Aviation Administration, Washington, DC.

[3] Bellis, Mary, "Early History of Flight," About.com Guide, http://inventors.about.com/library/ inventors/blearlyflight.htm, [retrieved 15 March 2012].

[4] Barrett, Betty, 2002, Aerospace Chronology 1896-1998, Labor Aerospace Research Agenda, Massachusetts Institute of Technology, Cambridge, MA.

[5] Scheck, William, March 2010, "The Development of the Autopilot," http://www.century-of-

flight.freeola.com/Aviation\%20history/evolution\%20 of\%20technology/autopilot.htm [retrieved 25 July 2012].

[6] "Greatest Engineering Achievements of the Twentieth Century," 2012, National Academy of Engineering, Washington, DC, [retrieved 15 March 2012].

[7] Derber, Alex, 24 June 2010, "Europe and USA take step towards ATM Interoperability", Flight International, England, United Kingdom.

[8] Boyle, Rebecca, 7 Feb. 2012, "Drones Will Be Admitted to Standard US Airspace By 2015", National Public Radio, Washington, DC, [retrieved 19 July 2012].

[9] Schutte, P. C., Goodrich, K. H., et al, 2007, The

Naturalistic Flight Deck System: An Integrated System Concept for Improved Single-Pilot Operations, NASA/TM-2007-21509. NASA:

Washington, DC.

[10] Dattee, Brice, 18 June 2007, "Challenging the SCurve: Patterns of Technological Substitution," DRUID Summer Conference 2007, Copenhagen, CBS, Denmark.

[11] Tushman, M. and P. Anderson, 1986, "Technological Discontinuities and Organizational Environments", Administrative Science Quarterly 31(3): 439-465.

[12] Booch, Grady, 15 Jan 2003, "The Limits of Technology”, IBM Software Group, [retrieved 12 July 2012].

[13] "NextGen Avionics Roadmap Version 2.0.", 30 Sept. 2011, Joint Planning and Development Office, Washington, DC. 
[14] Wise, Jeff, 9 July 2012, “Air France 447 and the Limits of Aviation Safety", Popular Mechanics, http://www.popularmechanics.com/technology/aviati on/crashes/air-france-447-and-the-limits-of-aviationsafety-10487501 [retrieved 12 July 2012].

[15] Perrow, C., 1984. Normal Accidents - Living with High Risk Technologies, New York, Basic Books.

[16] Webster CS, Stabile M, Merry AF, 2009, The challenges of technological intensification. APSF Newsletter, 24(3):33, 35, 43.

[17] Dolak, Kevin, 31 Aug. 2011, "Automation Addiction': Are Pilots Forgetting How to Fly?" ABC World News http://abcnews.go.com/Technology/ automation-addiction-pilots-forgetting-fly/story?id= 14417730\#.UBBLA7SXTng [retrieved 15 July 2012].

[18] Billings, C, August 1991, Human-Centered Aircraft Automation: A Concept and Guidelines, NASA TM 103885, Moffett Field, CA.

[19] Sterman, John D., 2000, Business Dynamics: Systems Thinking and Modeling for a Complex World, McGraw-Hill.

[20] Braun, W., 2002, "The System Archetypes", Washington, DC, http://wwwu.uni-klu.ac.at/gossimit/ pap/sd/wb_sysarch.pdf [retrieved 5 May 2010].
[21] Casner, S. M. (2002). Cockpit Automation for General Aviators and Future Airline Pilots. Ames, Iowa: Blackwell Publishing.

[22] Massie, M. J., and A. T. Morris, 29-31 March 2011, "Risk Acceptance Personality Paradigm: How We View What We Don't Know We Don't Know," AIAAInfotech@Aerospace Conference, St. Louis, Missouri.

[23] Morris, A. T., and Massie, M. J., 20-22 April 2010, "Analyzing Distributed Functions in an Integrated Hazard Analysis," AIAA Infotech@ Aerospace Conference, Atlanta, Georgia.

[24] Dion, R., July 1993, "Process Improvement and the Corporate Balance Sheet," IEEE transactions on Software Engineering, vol. 10, pp. 283-285.

[25] Hooks, Ivy F. and Farry, Kristin A., 2001, Customer-Centered Products: Creating Successful Products Through Smart Requirements Management, AMACOM.

[26] Joint Airspace Control, 20 May 2010, Joint Publication 3-52, United States Joint Forces Command, DOD, Washington, DC.

31st Digital Avionics Systems Conference October 14-18, 2012 